\title{
Recursive order-statistic soft morphological filters
}

\author{
S.-C.Pei \\ C.-L.Lai \\ F.Y.Shih
}

Indexing terms: Recursive order-statistic soft morphological filters, Morphological filtering, Image reconstruction

\begin{abstract}
A new class of recursive order-statistic soft morphological (ROSSM) filters are proposed and their important properties related to morphological filtering are developed. Criteria for specific selection of parameters are provided to achieve excellent performance in noise reduction and edge preservation. It is shown through experimental results that the ROSSM filters, compared to the order-statistic soft morphological filters or other well known nonlinear filters, have better outcomes in signal reconstruction. Two examples are given for demonstrating the flexibility of the proposed filters in signal processing applications.
\end{abstract}

\section{Introduction}

Mathematical morphology, a set-theoretical method, provides an approach to image processing based on geometrical shape of objects $[1,2]$. The principle applications of the morphological operations include signal digitisation, enhancement, compression, restoration, segmentation and description.

An important application in mathematical morphology is to develop an efficient morphological filter to restore an image from its noisy version. Schonfeld and Goutsias [3] presented a theoretic analysis of morphological filters for optimal restoration from noisy binary images. Parametric alternating sequential filters [4] are the popular choice to retrieve the important information which resides in an image and to eliminate the effect of noisy environment. Soft morphological (SM) filters $[5,6]$, the combination of morphological filters and order-statistic filters, have been shown to be less sensitive to additive noise and to small variations in object shape. Although Kuosmanen et al. [7, 8] discussed the optimisation and shape preservation of the SM filters, SM filters still have the disadvantage of lacking flexibility and applicability since the repetition times are set up to be the rank order. To overcome this drawback, Shih et al. [9] proposed an extended filtering class, order-statistic soft morphological (OSSM) filters,

(C) IEE, 1998

IEE Proceedings online no. 19982318

Paper first received 4th August and in revised form 4th December 1997 S.-C. Pei and C.-L. Lai are with the Department of Electrical Engineering, National Taiwan University, Taipei, Taiwan, Republic of China

F.Y. Shih is with the Department of Computer and Information Science, New Jersey Institute of Technology, Newark, NJ 07102, USA and showed that they have better performance in noise reduction and edge preservation while providing more flexibility in image applications.

In contrast to dealing with the filtering problems in nonrecursive transformations as stated above, another class of recursive transformations was introduced. Shih and Puttagunta [10] developed the recursive soft morphological (RSM) filters, their properties, cascade combinations and idempotent RSM filters. In general, recursive structures usually provide better smoothing capabilities and take less computational time even though this is at the expense of increased detailed distortion $[11,12]$.

In this paper, we develop recursive order-statistic soft morphological (ROSSM) filters to achieve better performance than before and offer more potential in developing various signal processing applications.

\section{Preliminary: recursive soft morphological filters and order-statistic soft morphological filters}

Recursive soft morphological (RSF) filters are those filters whose outputs depend not only on the original input signals but also on previously processed outputs within the window. That is, if $f_{i}$ and $y_{i}$ denote the input and output values at location $i$, respectively and the domain of the structuring element be $\{-L, \ldots,-1,0,1$, ... $R\}$, a recursive soft morphological filter on input $f$ can be defined as

$$
y_{i}=\Phi\left(y_{i-L}, \ldots, y_{i-1}, f_{i}, f_{i+1}, \ldots, f_{i+R}\right)
$$

where $\Phi$ is an usual soft morphological operation.

The order-statistic soft morphological (OSSM) operations adopt order-statistics to replace the maximum and minimum in the standard morphology and offer flexibility in free-order selection, and have been shown to be better in noise reduction and edge preservation than traditional nonlinear filters such as order-statistic filters (OSF), soft morphological filters (SMF) or median filters (MF) [9]. Moreover, the flexibility in selecting the order parameter makes them possible in many applications. However, the recursive structures, in general, have been shown to provide better smoothing capabilities and take less computational time even though this is at the expense of increased detailed distortion. A straightforward method to design filters which combines the advantages of OSSM and RSM filters is to join the structure of recursive and OSSM filters as a new filter structure, ROSSM filters, and is described in the following Section. 


\section{Recursive order-statistic soft morphological filters}

In this paper, to simplify the presentation, we concentrate on connected symmetric structuring elements. For other structuring elements, the corresponding results can be derived similarly. Moreover, the 2-D scanning order is also beyond our scope. To describe the recursive OSSM filters, the OSSM operations are defined first. Let $f$ denote a 1-D function and $(B, A),(A \subseteq B)$ denote the flat structuring element sets which are split subsets with the core set being $A$ and boundary set being $B \backslash A$, where " " denotes the set difference. Let the set translation of $A$ by vector $\mathrm{t}$ be defined as $(A)_{t}$, then the OSSM dilation (erosion) of $f$ by $[B, A, k, l]$ is defined as

$$
\begin{aligned}
(f \tilde{\oplus}(\tilde{\ominus}[B, A, k, l])(i) & =l \text {-th largest (smallest) of } \\
(\{k \diamond f(a) \mid a & \left.\left.\in(A)_{i}\right\} \bigcup\left\{f(b) \mid b \in(B \backslash A)_{i}\right\}\right)
\end{aligned}
$$

where $l$ represents the selected order and $k$ represents the repetition times of the elements within ' $(A)_{i}$ ' with $\{k$ $\diamond f(a)\}=\{f(a), f(a), \ldots, f(a)\}$ ( $k$ times).

To define the recursive OSSM filters, let $\Phi$ denote an ROSSM operation and $\left(B_{m}, A_{n}\right)$ be the symmetric structuring element sets with $m$ pixels in $B$ and $n$ pixels in $A$. For example, the structuring element $[-2,-1, \underline{0}, 1$, 2] is denoted as $B_{5} A_{1}$ while $[-2,-1,0,1,2]$ is denoted as $B_{5} A_{3}$ where the centre of the underscore denotes the centre of symmetry. Then, applying a recursive orderstatistic soft morphological filter on input $f$ at index $i$ can be written as

$$
y_{i}=\Phi\left(y_{i-L}, \ldots, y_{i-1}, f_{i}, f_{i+1}, \ldots, f_{i+R}\right)
$$

where $y_{i-L}, \ldots, y_{i-1}$ are already obtained by $\Phi\left(f_{i-L}\right), \ldots$, $\Phi\left(f_{i-1}\right)$.

The 2-D ROSSM operation on an image $F$ with structuring element $S$ is represented as

$$
Y_{i j}=\Phi\left(Y_{p}, F_{i j}, F_{q}\right)
$$

where $S=\left(S_{B}, S_{A}\right),\left(S_{A} \subseteq S_{B}\right)$, is a 2-D structuring element with core set $S_{A}$ and boundary set $S_{B} \backslash S_{A}$; and $p$, $q:(p \neq(i, j)$ and $q \neq(i, j))$ are domain indices sets that precede and succeed the index $(i, j)$ for some one scanning order (Fig. 1). An example is given to show the difference between the OSSM and ROSSM filters.
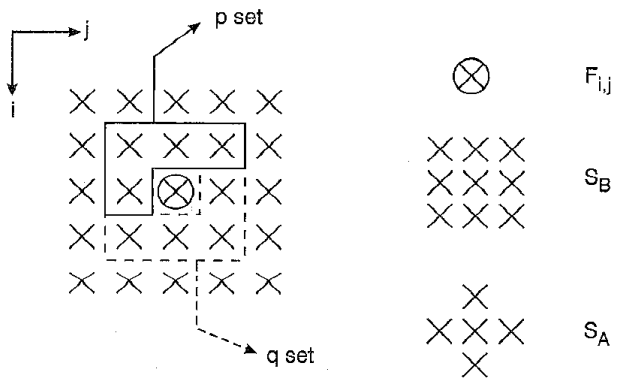

Fig. 1 2-D structuring element and the preceding (succeeding) domain indices sets when using the raster-scan order

Example 1: Let the structuring element be $B_{5} A_{1}$. Let $k$ $=2$ and $l=3$. Assume that the input sequence be $f=$ $\{74,14,28,65,55,8,61,20,17,65,26,94\}$ and the values which are outside the region of $f$ are set to be boundary values of $f$. Then the output sequences obtained from the OSSM and the ROSSM dilations are

$$
\begin{aligned}
\operatorname{OSSM} & (f, B, A, k, l) \\
& =\{74,65,55,55,55,55,55,20,26,65,65,94\}
\end{aligned}
$$

and

$$
\begin{aligned}
\operatorname{ROSSM} & (f, B, A, k, l) \\
= & \{74,65,65,65,61,61,61,61,61,65,65,94\}
\end{aligned}
$$

respectively. Figs. $2 a$ and $b$ show a $256 \times 256$ image and its disturbed version with additive Gaussian noise having zero mean and standard deviation of 20 . The reconstructed images by applying the OSSM and ROSSM filters with $S_{B}=[(-1,0),(0,-1),(0,0)(1,0)$, $(0,1)], \mathrm{S}_{A}=[(0,0)], k=3$ and $l=4$ are shown in Figs. $2 c$ and $d$ for visual comparison. The mean square signal-to-noise ratio, defined as

$$
S N R_{m s}=\frac{\sum\left(y_{i}^{\prime}\right)^{2}}{\sum\left(y_{i}^{\prime}-y_{i}\right)^{2}}
$$

with $y$ and $y^{\prime}$ denoting the reconstructed and original signal, respectively, is used to measure the fidelity of the reconstructed signals. The $S N R_{m s}$ values of Figs. $2 c$ and $d$ are 84.3 and 91.6, respectively. Moreover, it is observed that the visual perception effect of Fig. $2 d$ is better than Fig. $2 c$.

An important feature of the OSSM filter is the duality property [9]. This relationship property also holds for the ROSSM operators. That is, choosing $l$ in an ROSSM dilation is the same as choosing $T-l+1$ in an ROSSM erosion, where $T$ represents the total number in the order list. Let $\tilde{\oplus}_{R}$ and $\tilde{\Theta}_{R}$ denote the ROSSM dilation and erosion, respectively, then the duality relationship can be represented as $f \oplus_{R}[B, A, k$, $l]=f \tilde{\Theta}_{R}[B, A, k, T-l+1]$. However, if the structuring element is obtained by taking the intersection (union) of two or more structuring elements, this duality property may be unavailable.

\section{Properties of the ROSSM filters}

The basic properties related to the morphological filters are first discussed. According to the properties derived in [9], the OSSM filters are increasing, extensive (antiextensive) and possess the idempotent properties under some conditions which are referred in [13] since OSSM filters is a subclass of WOS filters. We will prove that the ROSSM filters are also increasing and extensive (anti-extensive). Before we discuss these properties, a basic property should be borne in mind that the first output signal value of an OSSM is the same as that of its corresponding ROSSM operator. It is trivial since the left-hand side domain of the first value are appended signals, as a result the full domains are the same. All the proofs of the developed properties are given in the Appendixes. The basic morphological properties are discussed as follows.

Property 1: The ROSSM filters are increasing.

Property 2: The ROSSM dilation is extensive and the ROSSM erosion is anti-extensive if $l \leq k$ and the origin of set $B$ belongs to set $A$.

Property 3: The idempotent ROSSM filters exist.

Although the exact expression of the idempotent ROSSM filters is not known, the idempotent ROSSM 


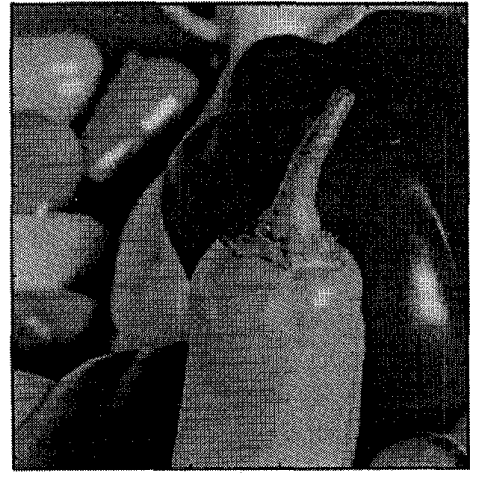

a

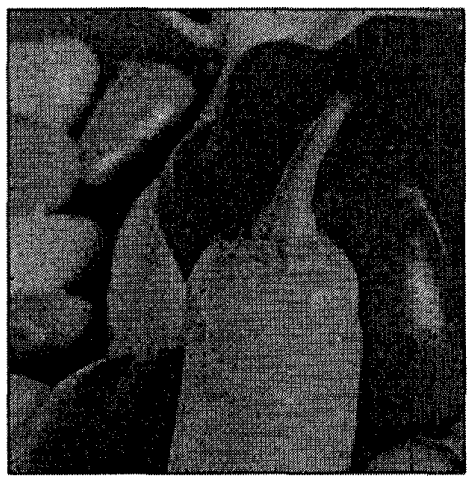

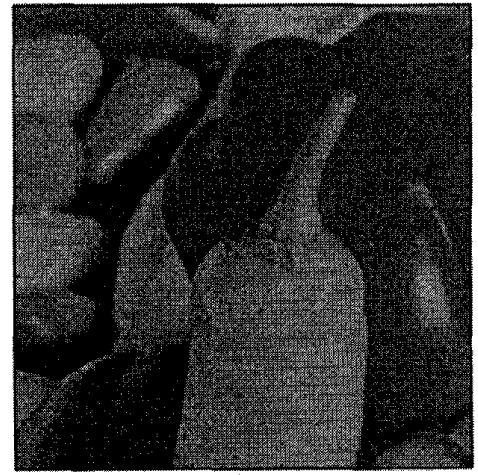

$b$

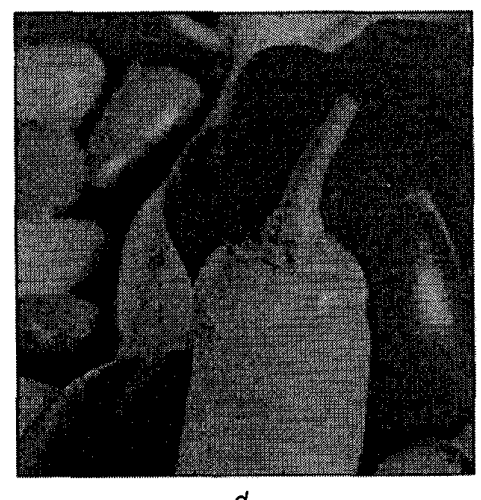

$d$

Fig.2 Visual comparison of the OSSM and ROSSM filters

$a$ Original image

$b$ Noisy image disturbed by zero mean Gaussian noise $(\sigma=20)$

Re OSSMF

filters do exist. For example, let the structuring element be a centre-weighted structure with size $2 N+1$, and let $k=2 N-1$ and $l=2 N$, then the resultant ROSSM dilation is idempotent. The identification of the exact form of the idempotent filter class is left to further work.

Example 2: Let the structuring element be $B_{5} A_{1}$. This implies that $N=2$. Let an input sequence $f=\{1,3,5$, $7,2,4,6,8,3,1,5,7,9\}$ and $k=3$ and $l=4$, then the first and second iterations of the ROSSM dilation output, denoted as $f^{1}$ and $f^{2}$, are $f^{1}=\{1,3,5,5,4,4,6,6$, $3,3,5,7,9\}$ and $f^{2}=\{1,3,5,5,4,4,6,6,3,3,5,7,9\}$, respectively, which states that this is an idempotent filter.

Properties 1-3 deal with the basic properties possessed by the morphological filters. According to property 3 and [2], a recursive morphological opening (closing) is obtained if the operator is idempotent and anti-extensive (extensive). In the following, some other: interesting properties relating to the ROSSM filters are developed.

Property 4: The ROSSM operator can be implemented by the threshold decomposition technique.

In the following we will discuss some distribution properties. Let $X$ and $Y$ denote the binary input signals, while $C$ and $D$ denote the structuring elements and $(k, l)$ be the parameters. Let the union/intersection of two structuring elements be defined as follows: The union (intersection) of two structuring elements is obtained by taking the maximal (minimal) range of the boundary and core sets of the original structuring ele-

ments. For example, $B_{7} A_{1} \cap B_{5} A_{3}=B_{5} A_{1}$ and $B_{7} A_{1} \cup$ $B_{5} A_{3}=B_{7} A_{3}$. Then we have

Property 5: $X \tilde{\oplus}_{R}(C \cap D) \subseteq\left(X \tilde{\oplus}_{R} C\right) \cap\left(X \tilde{\oplus}_{R} D\right)$

Property 6: $X \tilde{\oplus}_{R}(C \cup D) \supseteq\left(X \tilde{\oplus}_{R} C\right) \cup\left(X \tilde{\oplus}_{R} D\right)$

Property 7: $X \tilde{\Theta}_{R}(C \cap D) \supseteq\left(X \tilde{\Theta}_{R} C\right) \cap\left(X \tilde{\Theta}_{R} D\right)$

Property 8: $X \tilde{\Theta}_{R}(C \cup D) \subseteq\left(X \tilde{\Theta}_{R} C\right) \cup\left(X \tilde{\Theta}_{R} D\right)$

The foregoing four properties deal with the distribution from the right. For the distribution from the left, i.e. $(X \cap Y) \tilde{\oplus}_{R} C$, the same results can be obtained similarly.

Property 9: $(X \cap Y) \tilde{\oplus}_{R} C \subseteq\left(X \tilde{\oplus}_{R} C\right) \cap\left(Y \tilde{\oplus}_{R} C\right)$

Property 10: $(X \cup Y) \tilde{\oplus}_{R} C \supseteq\left(X \tilde{\oplus}_{R} C\right) \cup\left(Y \tilde{\oplus}_{R} C\right)$

Property 11: $(X \cap Y) \tilde{\Theta}_{R} C \supseteq\left(X \tilde{\Theta}_{R} C\right) \cap\left(Y \tilde{\Theta}_{R} C\right)$

Property 12: $(X \cup Y) \tilde{\Theta}_{R} C \subseteq\left(X \tilde{\Theta}_{R} C\right) \cup\left(Y \tilde{\Theta}_{R} C\right)$

Example 4: Assume that $X=\{1,0,1,1,1,1,0,0\}, C=$ $B_{7} A_{1}, D=B_{5} A_{3}, k=2$ and $l=3$. Then we have $C \cap D$ $=B_{5} A_{1}$ and $C \cup D=B_{7} A_{3}$. Let $Z, Z 0, Z 1$ and $Z 2$ denote the results of $X \tilde{\Theta}_{R}(C \cap D), X \tilde{\Theta}_{R}(C \cup D), X$ $\tilde{\Theta}_{R} C$, and $X \tilde{\Theta}_{R} D$, respectively. It is found that since $Z$ $=\{1,1,1,1,1,1,0,0\}, Z 0=\{1,1,1,1,1,0,0,0\}, Z 1$ $=\{1,1,1,1,1,0,0,0\}$, and $Z 2=\{1,1,1,1,1,0,0$, $0\}$, we have $Z \supseteq Z 1 \cap Z 2$ and $Z 0 \subseteq Z 1 \cup Z 2$, which is the result of property 7 and property 8 .

Up to now, we have discussed the case of binary signals. For gray scale signals, similar formulas can be obtained if $\cap, \cup, \subseteq$ and $\supseteq$ are replaced by min, $\max , \leq$ and $z$, respectively. The eight properties give a good way of predicting the upper and lower bounds of the filtering results when a ROSSM operation with multi- 
ple input signals or structuring elements is performed, which is helpful in some image analysis applications. In the following, an associativity feature phenomenon is concerned. To state this phenomenon, a new product operation $\odot$ of two structuring elements is defined as multiplying the weighted values of the components by each other and then combining the results by addition. For example, if two structuring elements $\left(C=B_{3} A_{1}, k\right.$ $=2)$, and ( $\left.D=B_{3} A_{1}, k=3\right)$ are given, then $C \odot D$ can be rewritten as $[1, \underline{2}, 1] \odot[1, \underline{3}, 1]$ and is computed as $[1,5, \underline{8}, 5,1]$. That is, it could be viewed by taking a normal multiplication of two numbers, which are obtained by expanding all the weighted components, and regarding each digit as a weighting value as shown in Fig. 3. Then the associativity phenomenon can be observed as

Phenomenon 1: $X \tilde{\oplus}_{R} C \tilde{\oplus}_{R} D \subseteq W O S(X,(C \odot D))$ where $W O S(X, M)$ denotes a weight order-statistic filtering with input $X$ and weighting coefficients matrix $M$.

$$
\begin{aligned}
& \underset{B_{3} A_{1}}{{ }_{k=2}} \odot \begin{array}{l}
B_{3} A_{1} \\
k=3
\end{array} \\
& {[1 \underline{2} 1] \odot[1 \underline{3} 1]=\frac{12 \underline{1} 1}{15 \underline{3} \underline{s} 1}}
\end{aligned}
$$

Fig.3 Product operation of two structuring elements

An example is given to try to derive this phenomenon; other cases with different parameters could be derived similarly. Let $\left(C=B_{3} A_{1}, k=2\right)$ and ( $D=B_{3} A_{1}$, $k=2)$. Thus, $C \odot D=[1, \underline{2}, 1] \odot[1, \underline{2}, 1]=[1,4, \underline{6}, 4$, 1]. Let $Y=X \tilde{\oplus}_{R} C, Z=Y \tilde{\oplus}_{R} D$ and $R=\operatorname{WOS}(X,[1,4$, $\underline{6}, 4,1])$. According to [13], it is found that

$$
\begin{aligned}
y_{i-1} & =\text { Boolean }\left(x_{i-2}+2 x_{i-1}+x_{i} \geq l\right) \\
y_{i} & =\operatorname{Boolean}\left(x_{i-1}+2 x_{i}+x_{i+1} \geq l\right) \\
y_{i+1} & =\text { Boolean }\left(x_{i}+2 x_{i+1}+x_{i+2} \geq l\right) \\
z_{i} & =\operatorname{Boolean}\left(y_{i-1}+2 y_{i}+y_{i+1} \geq l\right) \\
r_{i} & =\operatorname{Boolean}\left(x_{i-2}+4 x_{i-1}+6 x_{i}\right. \\
& \left.\quad+4 x_{i+1}+x_{i+2} \geq l\right)
\end{aligned}
$$

It is noted that if $z_{i}=1$, there must exist at least one one-value point, for example $y_{i}$, such that its corresponding expression, $\left(x_{i-1}+2 x_{i}+x_{i+1} \geq l\right)$ holds. Since $x_{i} \geq 0$, it is easy to find that $\left(x_{i-2}+4 x_{i-1}+6 x_{i}+4 x_{i+1}+\right.$ $x_{i+2} \geq l$ ) is also satisfied. As a result, we have $r_{i}=1$, which leads to the result of this phenomenon.

Example 5: Let $f=\{29,54,51,10,41,58,88,44\}, C=$ $D=B_{3} A_{1}, k=2$ and $l=3$. It is observed that $f \tilde{\oplus}_{R} C \tilde{\oplus}_{R} D=\{29,51,51,10,41,58,58,44\}$, which is smaller than (bounded by) the result of $\operatorname{WOS}(f$, $[14 \underline{6} 41])=\{54,54,54,51,58,88,88,88\}$.

The associativity phenomenon has its applicability in analysing the cascade behaviour of two ROSSM filters. If an input signal undergoes two ROSSM filtering processes with two arbitrary structuring elements, it is possible to predict an upper bound of the output signal by applying a WOS filter. That is, it is possible to use a one-pass filtering process to approximate a filtering of two or more passes and this is helpful to many applications. However, it should be noted that a similar procedure for erosion to predict a lower bound is not yet available.

\section{Experimental results and discussions}

In this Section, computer simulations are performed to validate the good performance of the ROSSM filters. To compare the performance with the OSSM filters in edge preservation and noise reduction, three noise models, Gaussian, uniform and Pepper-salt are adopted for comparison. The input 1-D signal and the corrupted signals corresponding to the three noise models are shown in Fig. 4. Both the 'mean square SNR value', which was defined previously, and the 'transition rate' are used to assess the performance in noise reduction and edge preservation, respectively.
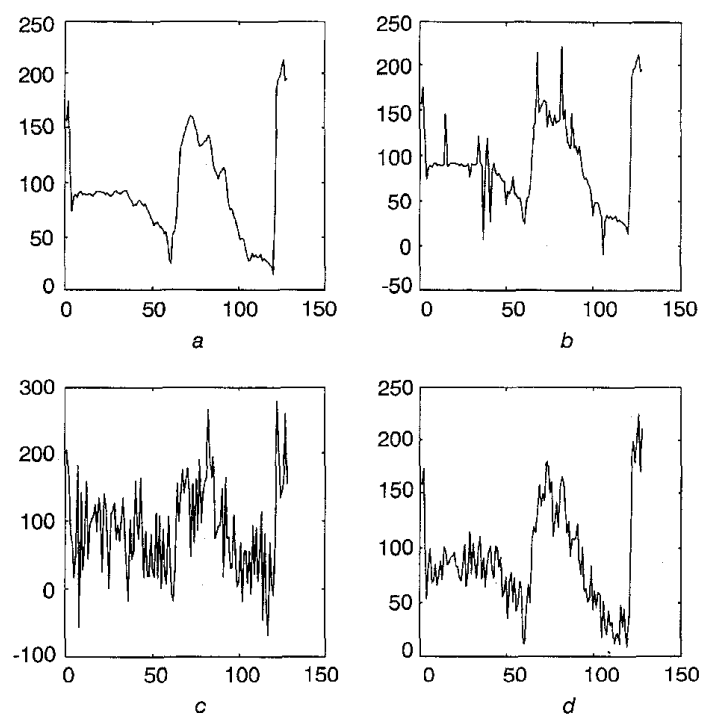

Fig. $4 \quad 1-D$ original signal and its noise-corrupted versions a Original signal

$b$ Corrupted by Gaussian noise (mean $=0$ and $\sigma=50$ ) $c$ Corrupted by pepper-salt noise (occurrence probability $P_{o}=0.2$ and standard deviation $\sigma=50$ )

$\mathrm{d}$ Corrupted by uniform distributed noise (mean $=0$ and $\sigma=50$ )

To define the transition rate, a test method stated in [14] is adopted and is described as follows. A step edge signal $f$, denoted as $f(i)=100(i<128) ; f(i)=200(i \geq$ 128 ) is corrupted by the given three noise models separately and its ROSSM filtering output is denoted as $y$. The transition rate $T$, defined as the percentage of edge reconstruction from its disturbed version, is represented as

$$
T=1-\frac{|E[y(128)]-E[y(127)]|}{100}
$$

where $E[y(i)]$ is estimated by averaging 128 different trial outputs. Therefore, it can be used as the performance meter of edge preservation, where value ' 1 ' represents 'perfect' preservation. Figs. 5-7 show the mean square SNR values corresponding to three different noise models while Figs. 8-10 show their transition rate results. From these results, it is found that for Gaussian and Pepper-salt noise models, the $\mathrm{SNR}_{m s}$ values of the ROSSM filters are almost greater than those of the OSSM filters. However, the transition rates of the ROSSM and OSSM filters are nearly the same for all three noise models. In image processing applications, since images are often corrupted by natural noise (Gaussian) or impulses stemming from decoding errors or noisy channels, and both are easily detected by eye and degrade the image quality, it is observed that the 

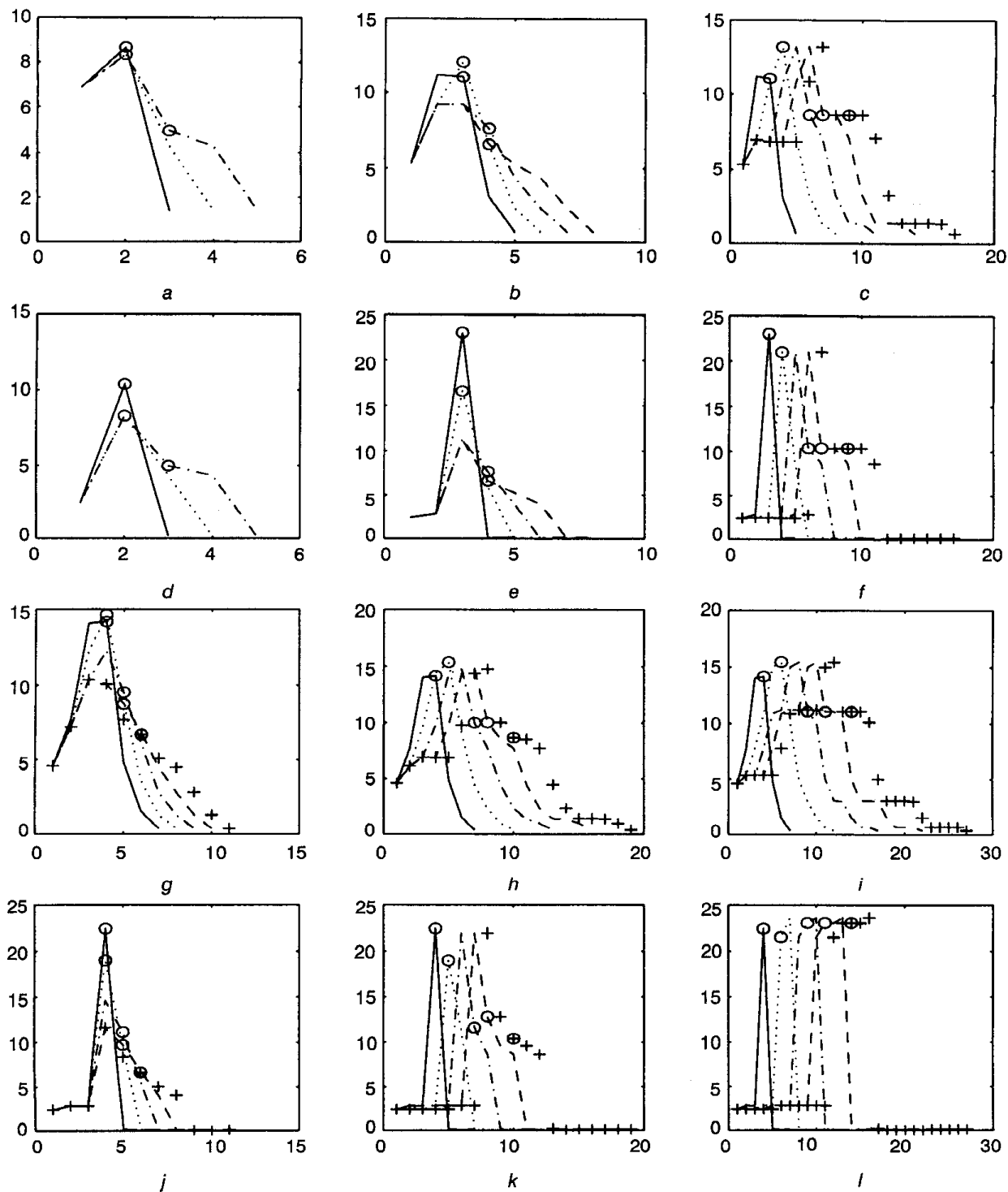

Fig.5 SNR Gaussian distributed noise by different structuring elements $a-c, g-i$ OSSM dilation
$d-f, j-l$ ROSSM dilation

$a, d B_{3} A_{1} ; \mathrm{b}, e B_{5} A_{1} ; c, f B_{5} A_{3} ; g, j B_{7} A_{1} ; h, k B_{7} A_{3} ; i, l B_{7} A_{5}$

$a, d B_{3} A_{1} ; \mathrm{b}, e B_{5} A_{1} ; c, f B_{5} A_{3} ; g, j B_{7} A_{1} ; h, k$
$---k=1$ (OSF)

$\begin{array}{llll}\cdots \cdots . . & k=2 & + & k=5 \\ \cdots \cdots \cdots & k=3 & 0 & \text { median filter }\end{array}$

proposed ROSSM filters are better in noise reduction applications while maintaining the advantage of edge preservation as in the OSSM filter. Since OSSM filters is a subclass of WOS filters, many well known nonlinear filters, such as MF, OSF and SMF, can be represented by the OSSM filters with different $(k, l)$ parameters [9]. As a result, we can conclude that the ROSSM filter is better than those nonlinear filters.

It should be noted that the analysis of optimisation in using the ROSSM filters is nearly the same as in the OSSM filters. However, by observing Figs. 5-10, the $l$ ranges that can be used in the ROSSM filters are somewhat narrower than those of the OSSM filters. It means that it is a little more strict in selecting a suitable $l$ value when the ROSSM filters are used in noise reduction applications. Moreover, the cascade behaviour of two ROSSM filters is similar to the analysis in
[10]. Although no direct formulas which can describe the combination of any two ROSSM filters exactly are available, the analysis gives the principal idea in constructing the combined filter from two different filters.

To illustrate the flexibility of using the ROSSM filters in image processing, two examples, one for impulse noise elimination and the other for ramp edge enhancement, are developed. The first example deals with the problem of reconstructing signals from impulse noise environment and is stated as follows. Assume that an original flat signal, shown in Figs. $11 a, e, l$ and $m$ is corrupted by impulses (positive or negative) as shown in Figs. $11 b, f, j$ and $n$. To eliminate the impulse noise, an OSSM dilation with $B=B_{3} A_{1}, k=1$ and $l=2$ is applied and the result are shown in Figs. $11 c, g, k$ and $o$. It is found that the impulse noise is not completely removed. However, if a ROSSM dilation with the same 

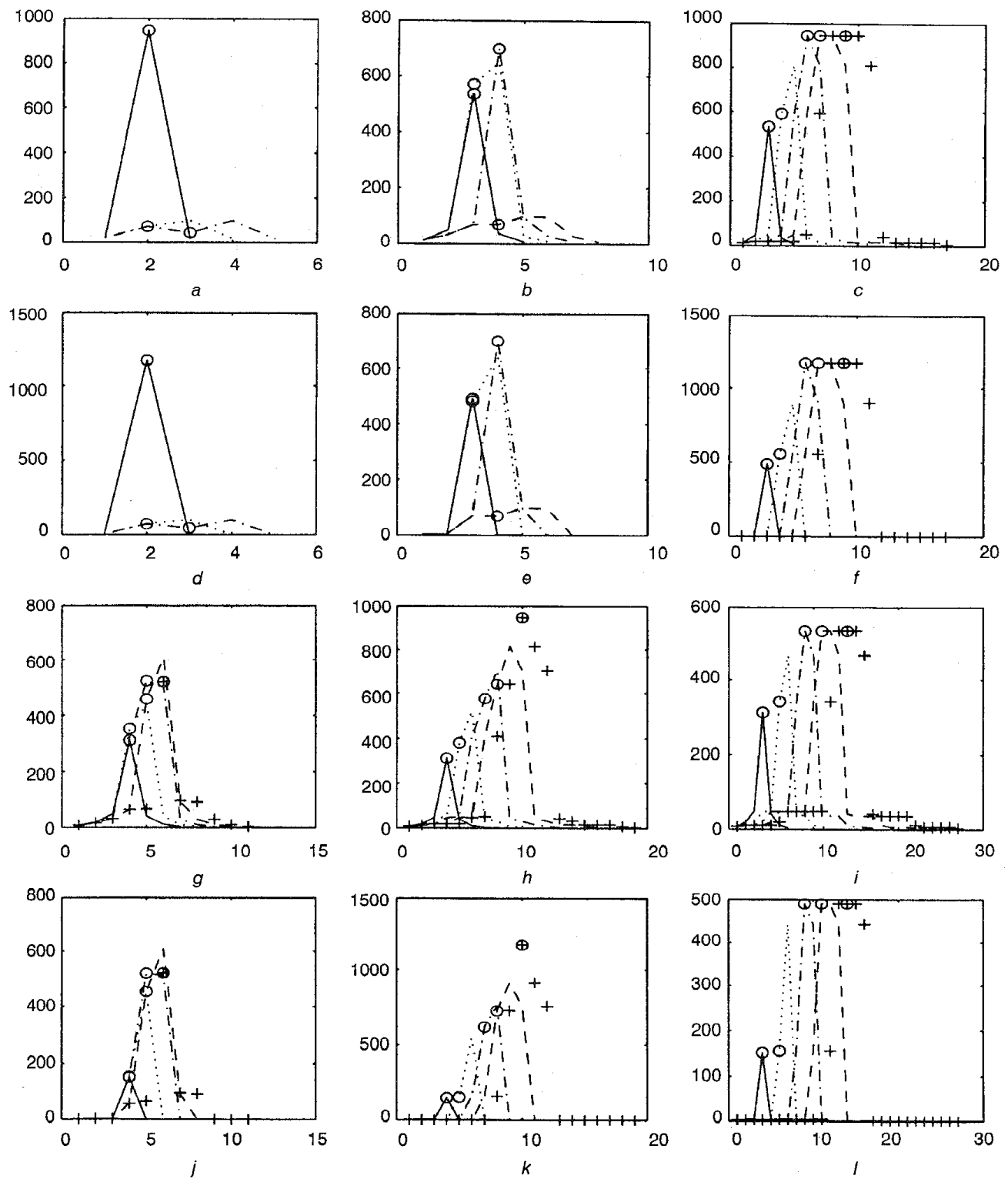

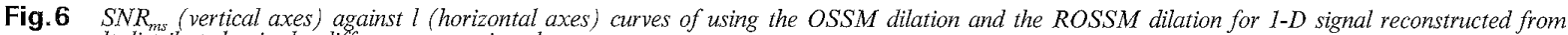
pepper-salt distributed noise by different structuring elements $a-c, g-i$ OSSM dilation $a, d B_{3} A_{1} ; b, e B_{5} A_{1} ; c, f B_{5} A_{3} ; g, j B_{7} A_{1} ; h, k B_{7} A_{3} ; i, l B_{7} A_{5}$.

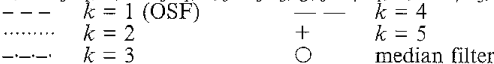

parameters is used, the original signal can be reconstructed perfectly (except the result shown in Fig. 11h) as shown in Figs. 11d, $l$ and $p$. It should be noted that although we cannot make a strong conclusion that the ROSSMF is better than OSSMF in impulse removing just by the foregoing examples, we can expect that the ROSSMF is better in impulse reduction in most cases.

In the second example, an edge enhancement problem is given since it is important for feature detection or visual perception in image processing. Owing to the flexibility of the ROSSM filters, the ramp edges can be transformed to step edges, as shown in Figs. 12 and 13, by using a 1-D ROSSM filter. The algorithm is described as follows. Let a structuring element be $B_{5} A_{1}$ and $k=1$ for simplicity. The input image $f$ of size $M \times$
$N$ is first decomposed into $M$ row sequences, which has $N$ pixels in each. For each point $f_{m}(i)$ of the sequence $f_{m}$, its tangent $T a$ is first determined through $f_{m}(i)-f_{m}(i$

- 1), then $l$ is chosen according to the tangent value. If $T a$ is greater than a threshold value $T h=12, l$ is set to 4. If $T a$ is smaller than $-T h, l$ is set to 2. Those cases with $T a$ ranging within $[-T h, T h]$ will set $l$ to 3 . The threshold value $T h$ controls the sensitivity of the ramp edge detection and varies according to different applications. By applying this ROSSM filter, a blurred Lena image, shown in Fig. 13a, can be enhanced as shown in Fig. 13b. It is noted that the blurred edges are now enhanced as clear boundaries. An additional advantage is also observed in the Figure that after being processed by this ROSSM filter, the textures, sometimes defined 

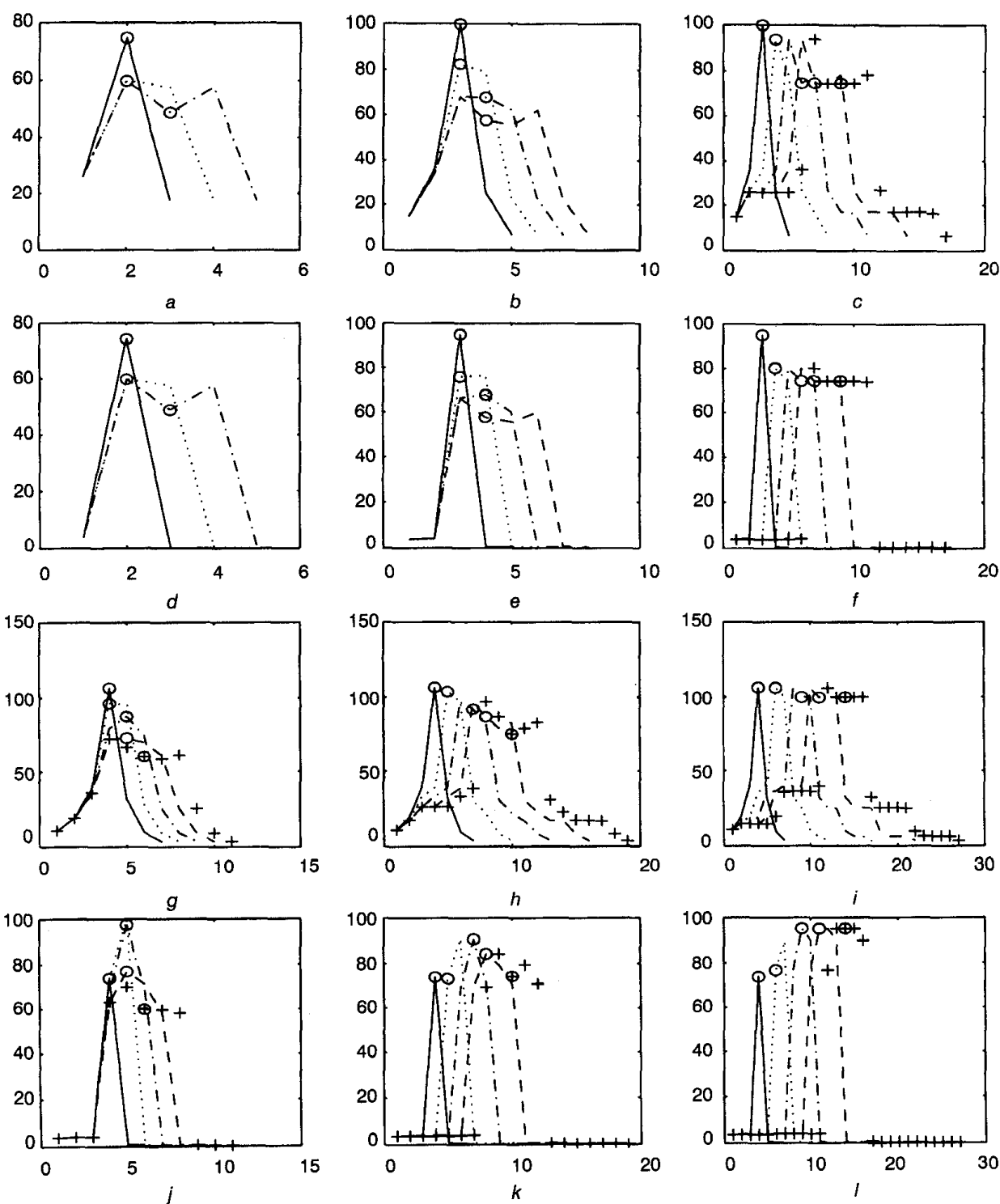

Fig.7 SNR $R_{m y}$ (vertical axes) against l (horizontal axes) curves of using the OSSM dilation and the ROSSM dilation for 1-D signal reconstructed from uniform distributed noise by different structuring elements $a-c, g-i$ OSSM dilation
$d-f, j-l$ ROSSM dilation

$a, d B_{3} A_{1} ; b, e B_{5} A_{1} ; c, f B_{5} A_{3} ; g, j B_{7} A_{1} ; h, k B_{7} A_{3} ; i, l B_{7} A_{5}$.

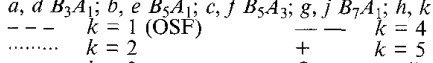

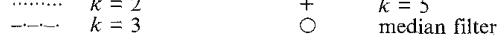

by pseudoperiodic patterns, are suppressed while the edges are enhanced. This leads to some other applications which need to separate edges from textures. It should be noted that since the 1-D procedure is used to process the Lena image, Lena's hat and hand are quite distorted. This scratched effect, however, may be erased by using a suitable 2-D directional morphological filter which is not yet available. For the 1-D signal as shown in Fig. 12a, the above procedure can be applied to obtain the result of Fig. $12 b$.

The solution of optimal selection of parameters $(k, l)$ for general uses is still not on hand since each application may need a specific parameter of its own. However, according to this work, readers can choose suitable parameters when dealing with applications of noise reduction, edge preservation and edge enhance- ment. To sum up, the proposed ROSSM filters not only support a flexible structure in developing enhanced signal processing applications, but also have better performance in noise reduction when the noise is either of Gaussian or pepper-salt distribution. That is, both advantages of using the recursive filters and the OSSM filters are preserved in the ROSSM filtering structure.

\section{Conclusions}

This paper introduces an extended class of morphological filters, ROSSM filters, for signal processing applications. The ROSSM filters, like the OSSM filters, offer flexibility in designing filters for various applications and efficiency in noisy images reconstruction. 

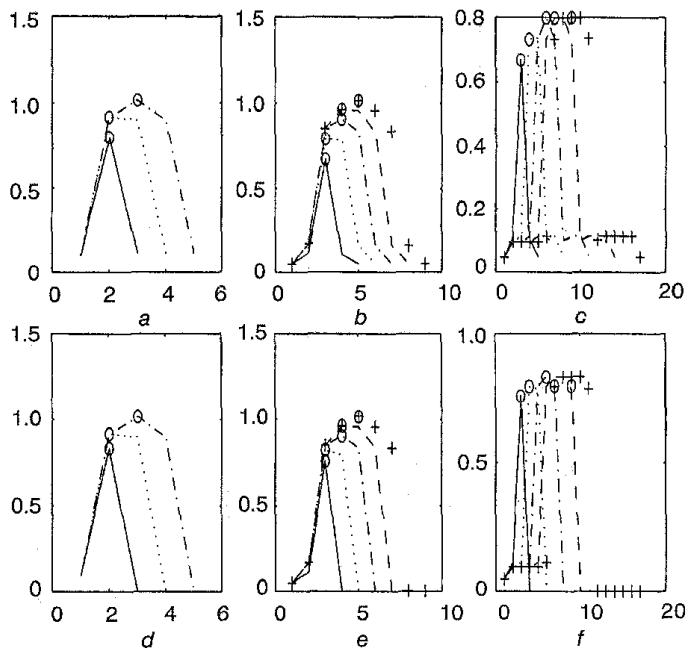

Fig. 8 Transition rate (vertical axes) against $l$ (horizontal axes) curves of using the OSSM dilation and the ROSSM dilation for reconstructing a step edge disturbed by Gaussian noise Different structuring elements are used $a-c$ OSSM dilation

$d-f$ ROSSM dilation

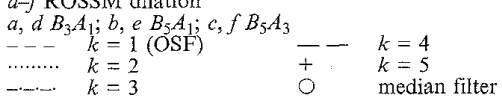
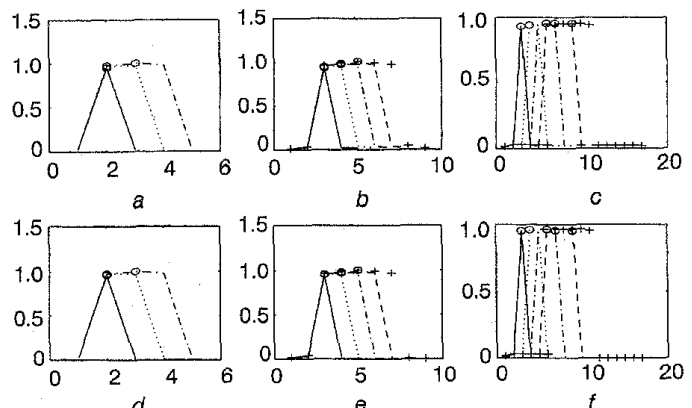

Fig.9 Transition rate (vertical axes) against $l$ (horizontal axes) curves of using the OSSM dilation and the ROSSM dilation for reconstructing $a$ step edge disturbed by pepper-salt distributed noise

Different structuring elements are used

$a-c$ OSSM dilation

$d-f$ ROSSM dilation

a, $d B_{3} A_{1} ; b$, e $B_{5} A_{1} ; c, f B_{5} A_{3}$

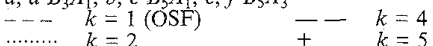

$\begin{array}{llll}\ldots . . . & k=2 & + & k=5 \\ \text { _.... } & k=3 & 0 & \text { median filter }\end{array}$
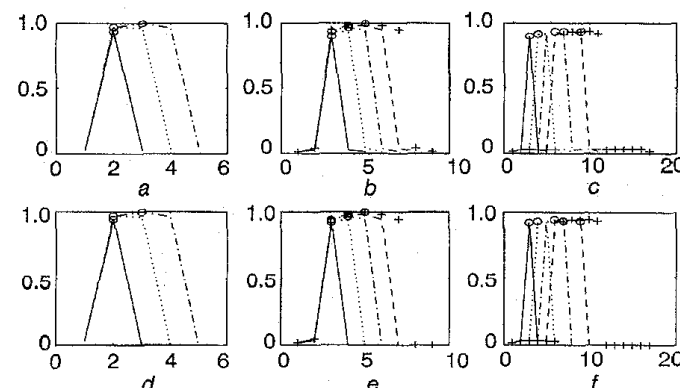

Fig. 10 Transition rate (vertical axes) against. $l$ (horizontal axes) curves of using the OSSM dilation and the ROSSM dilation for reconstructing a step edge disturbed by uniform distributed noise

Different structuring elements are used

$a-c$ OSSM dilation

$d-f$ ROSSM dilation
$a, d B_{3} A_{1} ; b, e B_{5} A_{1} ; c, f B_{5} A_{3}$

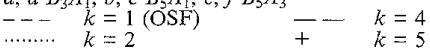

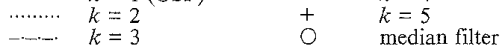

340
Some important properties related to the morphological filters are derived and the extensive properties of the proposed filters are developed. Comparing the experimental results with OSSM filters (equivalent to comparing with many well known nonlinear filters), it is observed that the ROSSM filters have better performance in noise reduction when the noise is either Gaussian or pepper-salt distributed, which covers most cases in image processing. However, the edge preservation feature in the OSSM filters is also maintained. Two applications, ramp edge enhancement and impulse noise elimination, are also presented to provide the flexibility feature of the proposed ROSSM filters. As a result, it is concluded that the ROSSM filters preserve those benefits of OSSM filters and recursive filters and are suitable for enhanced signal processing applications.

\section{Acknowledgments}

This research was supported by the National Science Council of the Republic of China under NSC 85-2213E-002-010.

\section{References}

1 MATHERON, G.: 'Random sets and integral geometry' (Wiley, New York, 1975)

2 SERRA, J.: 'Image analysis and mathematical morphology' (Academic, New York, 1982)

3 SCHONFELD, D., and GOUTSIAS, J.: 'Optimal morphological pattern restoration from noisy binary image', IEEE Trans Patt. Anal. Mach. Intell., 1991, 13, (1), pp. 14-29

4 STERNBERG, S.R.: 'Grayscale morphology', Comput. Vis. Graph. Image Process., 1986,35 , pp. 333-355

5 KOSKINEN, L., ASTOLA, J., and NEUVO, Y.: 'Soft morphological filters', Proc. SPIE - Int. Soc. Opt. Eng., 1991, 1568, pp. $262-270$

6 KOSKINEN, L., and ASTOLA, J.: 'Statistical properties of soft morphological filters', Proc. SPIE - Int. Soc. Opt. Eng., 1992, 1658 , pp. $25-36$

7 KUOSMANEN, P., and ASTOLA, J.: 'Soft morphological filtering', J. Math. Imaging Vis., 1995, 5, (3), pp. 234-262

8 KUOSMANEN, P., KOIVISTO, P., HUTTUNEN, H., and ASTOLA, J.: 'Shape preservation criteria and optimal soft morphological filtering', J. Math. Imaging Vis., 1995, 5, (4), pp. $319-$ 335

9 SHIH, F.Y, LAI, C.L, PEI, S.C, and HORNG, J.H. 'Order statistic soft morphological filters'. 3rd International conference on Computer science and informatics, America, March 1997

10 SHIH, F.Y., and PUTTAGUNTA, P.: 'Recursive soft morphological filters', IEEE Trans. Image Process., 1995, 4, (7), pp. $1027-1032$

11 HARALICK, R.M., and SHAPIRO, L.G.: 'Computer and robot vision’ (Addison-Wesley, 1992), pp. 57-126

12 STERNBERG, S.R., and STERNBERG, E.S.: 'Industrial inspection by morphological virtual gauging'. IEEE Workshop on Comput. architecture pattern anal. image database management, Oct. 1983 , pp. $237-247$

13 YU, P.T., and LIAO, W.H.: "Weighted order statistics filters Their classification, some properties, and conversion algorithm', IEEE Trans. Signal Process., 1994, 42, (10), pp. 2678-2691

14 YOU, $X$., and CREBBIN, G.: 'A robust adaptive estimation for filtering noise in images', IEEE Trans. Image Process., 1995, 4 (5), pp. 693-699

\section{Appendixes}

To complete the proofs of this paper, the following notations are introduced. Let $X$ and $Y$ denote the binary input sequence; $C, D$ and $W$ denote the binary structuring element (thus they can be viewed as the weighting coefficient $[9,13]$ ); while $Z, Z 1$ and $Z 2$ denote the binary output sequence. The $i$ th component of a particular sequence, for example $Z$, is denoted as $z_{i}$.

IEE Proc-Vis. Image Signal Process., Vol. 145, No. 5, October 1998 

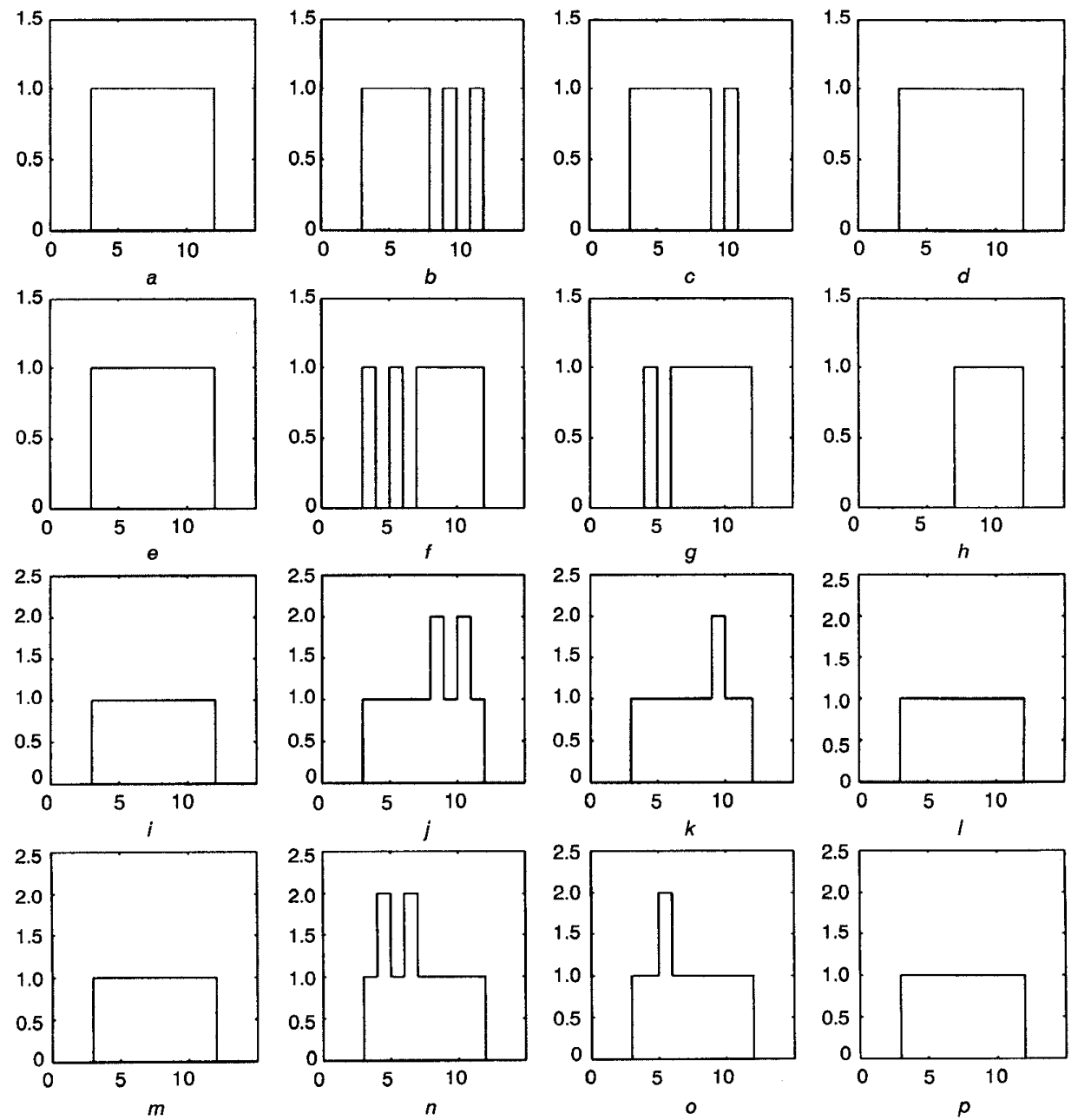

Fig.11 Signal reconstruction examples

$\begin{array}{ll}a, e, i, m & \text { Original signals } \\ b, f, j, n & \text { Their disturbed versions by impulse noise }\end{array}$

$c, g, k, o \quad$ Reconstructed signals from OSSM filter

$d, h, l, p \quad$ Reconstructed by ROSSM filter

Note that $B=B_{3} A_{1}, k=1$, and $l=2$

\subsection{Proof of property 1}

This is a trivial consequence of the fact that ROSSM filters are recursive weighted order statistic (WOS) filters and thus a subclass of stack filters, which in turn are increasing by the definition of the positive Boolean functions.

\subsection{Proof of property 2}

This property can be proved by intuition as follows. Since an OSSM dilation on $f$ at location $i, y=$ $\operatorname{OSSM}(f, B, A, k, l)(i)$ is to choose the $l$ th largest value from $f$ within domain $B_{i}$, we can write the descending sort list as $\left[f_{U}, k \diamond f(i), f_{L}\right]$, where $f_{U}=\left\{f_{U 1}, f_{U 2}, \ldots\right.$, $\left.f_{U m}\right\}$ and $f_{L}=\left\{f_{L 1}, f_{L 2}, \ldots, f_{L n}\right\}$ represents upper (greater than $f(i)$ ) and lower part sets, respectively. It is found that as long as $l \leq k$ is satisfied, either a greater value $f_{U j}$ in $f_{U}$ or an equivalent value of $f(i)$ will be picked out. Thus, we always have $f \leq O S S M(f), \forall i$. Therefore, for the input domain of the ROSSM dilation, there must exist at least one point $y_{i-1} \geq f_{i-1}$ such that the upper part of the new sort list $\left[f_{U}^{\prime}, k \diamond f(i), f_{L}^{\prime}\right]$ is always greater than $f_{U}$ both in its domain and range. Thus, from property 1 , it is concluded that the ROSSM dilation is extensive. Similarly, the proof of the anti-

extensive property of a ROSSM erosion can be obtained immediately when the duality relationship is used.

\subsection{Proof of property 4}

It is trivial, cf. the proof for property 1 .

\subsection{Proof of property 5}

We first prove this property for binary OSSM filters since gray results can be obtained by using property 4 . Let $W=C \cap D$ while $Z, Z 1$ and $Z 2$ be the results of $X \tilde{\oplus}(C \cap D), X \tilde{\oplus} C$ and $X \tilde{\oplus} D$, respectively. According to the theorem in [13], for each $z_{i}=1$ we always have the following equation:

$$
\sum^{L} w_{i} x_{i} \geq l
$$

where $w_{i} x_{i}$ represents the 'AND' operation of $w_{i}$ and $x_{i}$, where $x$ is the input data block obtained by shifting the central point of $W$ to position $i$ and takes the values within the domain $L$ of the structuring element. It is observed that since $W \subseteq C$ and $W \subseteq D$, we always have $\Sigma w_{i} x_{i} \leq \Sigma c_{i} x_{i}$ and $\Sigma w_{i} x_{i} \leq \Sigma d_{i} x_{i}$. It leads to the result that if $z_{i}=1$, then $z_{i}^{1}=1$ and $z_{i}^{2}=1$ also hold, which leads to the results $X \tilde{\oplus}(C \cap D) \subseteq(X \tilde{\oplus} C) \cap(X \tilde{\oplus} D)$. 

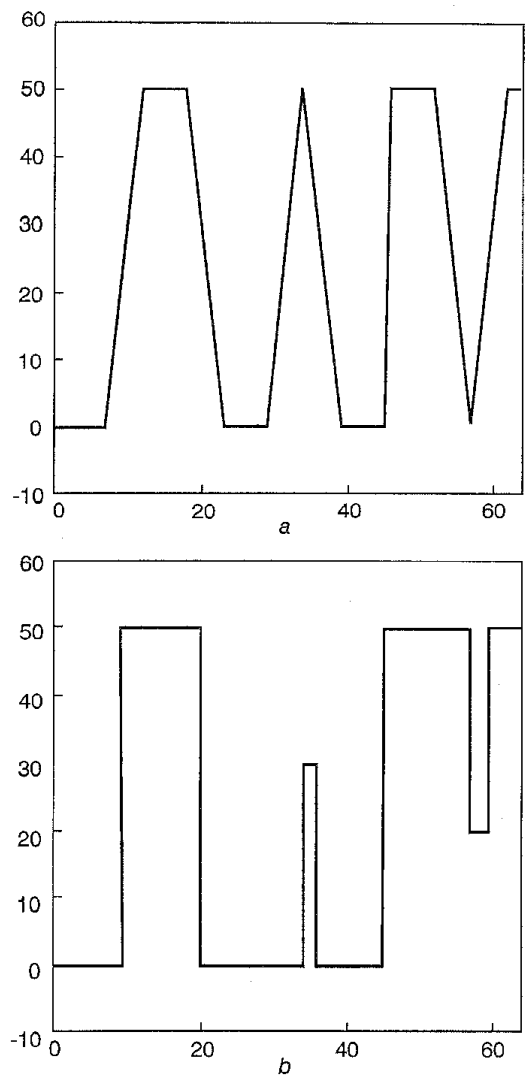

Fig. $12 \quad l-D$ edge enhancement example $a$ Original signal

Thus, if the input $x_{i}$ is replaced by the recursive output $\Phi\left(x_{i}\right)$, the same result can be immediately obtained for the ROSSM filters.

\subsection{Proof of property 6}

This proof is similar to the above thus only the OSSMF part is given. Because $W=C \cup D$, we always
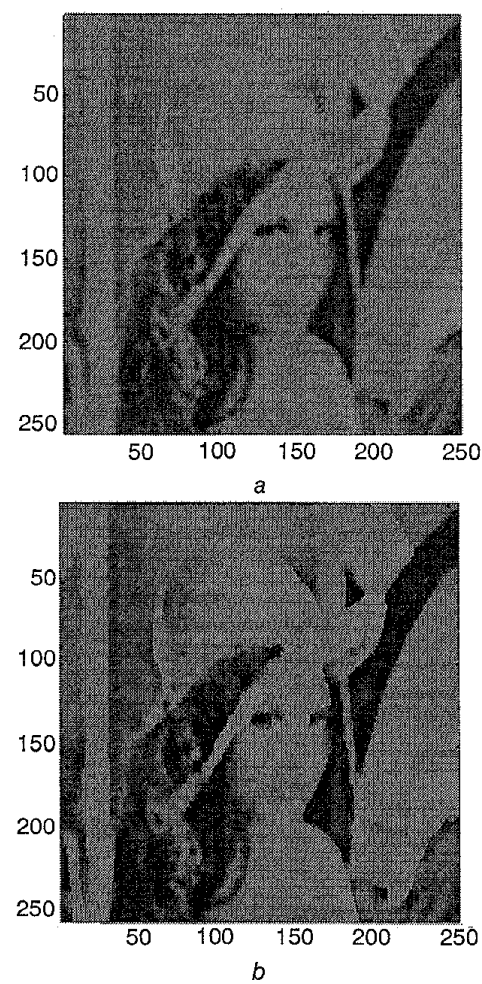

Fig. 13 2-D image edge enhancement example $a$ Original image

have $\Sigma w_{i} x_{i} \geq \sum c_{i} x_{i}$ and $\Sigma w_{i} x_{i} \geq \sum d_{i} x_{i}$. As a result, if $z_{i}^{1}=$ 1 or $z_{i}^{2}=1$, i.e. $\Sigma c_{i} x_{i} \geq l$ or $\Sigma d_{i} x_{i} \geq l$, it is also true that $z_{i}=1$, which leads to the equation of this property.

\subsection{Proof of property 7}

According to [13]. An output point $z_{i}$ is set to ' 1 ' if $\Sigma w_{i} x_{i} \leq l$. Since $\Sigma w_{i} x_{i} \leq \Sigma c_{i} x_{i}$ and $\Sigma w_{i} x_{i} \leq \Sigma d_{i} x_{i}$, it is observed that if $z_{i}^{1}=1$ and/or $z_{i}^{2}=1$, we always have $z_{i}=1$. Proofs of properties $8-12$ are omitted since they can be easily derived by using similar methods. 